\title{
Cumplimiento sin Cumplimiento La UIF-Perú incorporada a la SBS, oficial sin autonomía, la función a dedicación no exclusiva: una ventana hacia el lavado de activos
}

\author{
Daniel Ángel Puémape Postigo
}

Abogado por la Universidad de San Martín de Porres. Estudios en Derecho Bancario en la Universidad De La Sabana de Colombia.

SUMARIO:

I. Introducción.

II. La UIF-Perú adscrita a la SBS.

III. La dedicación no exclusiva.

IV. Supuesta autonomía.

V. Anticipación del directorio.

VI. Remoción del oficial de cumplimiento.

VII. Informes confidenciales y reservados.

VIII. Reflexiones finales. 


\section{RESUMEN:}

En el presente artículo, el autor señala tajantemente las deficiencias legales del sistema de prevención de lavado de activos: las deficiencias normativas y competencia de los entes reguladores que han contribuido a la imperfección para fortalecer el sistema de prevención, la poca autonomía funcional de la UIF-Perú incorporada a la SBS en el rol de sus funciones, la dedicación no exclusiva y la falta de autonomía del oficial de cumplimiento, la falta de prevención y voluntad de control de los dueños y directivos prontos a asumir riesgos y consecuencias penales en la organización, y la reserva de los informes confidenciales son algunas de las deficiencias hacia una ventana del lavado de activos.

Palabras clave: cumplimiento, prevención, lavado de activos, UIF-Perú.

\footnotetext{
ABSTRACT:

In this article, the author points out the legal deficiencies of the money laundering prevention system: the regulatory deficiencies and competence of the regulatory entities that have contributed to the imperfection to strengthen the prevention system, the little functional autonomy of the UIF-Peru incorporated to the SBS in the role of its functions, the non-exclusive dedication and lack of autonomy of the compliance officer, the lack of prevention and control willingness of shareholders and managers ready to assume risks and criminal consequences in the organization, and the confidentiality of reserved reports are some of the deficiencies towards a money laundering window.

Keywords: compliance, prevention, money laundering, UIF.
}

\section{INTRODUCCIÓN}

El lavado de activos es un fenómeno de dimensiones internacionales. Dada la interdependencia existente entre los países y el alto desarrollo tecnológico de las telecomunicaciones, los capitales fluyen fácilmente por el sistema financiero, bancario y bursátil a nivel mundial. Esto permite a la delincuencia organizada operar local e internacionalmente, por lo cual el lavado de activos normalmente involucra movimiento de capitales entre distintos países.

De allí la necesidad de cooperar internacionalmente con estándares de supervisión y regulación idónea, cooperación que ha sido reconocida por numerosos gobiernos y ha redundado en diversos acuerdos en el ámbito internacional. En buena medida, estos acuerdos han contribuido a fortalecer los ordenamientos nacionales y mejorar los mecanismos de cooperación e intercambio de información y pruebas en diversos países.

Puede afirmarse que, en el ámbito nivel latinoamericano, los mecanismos de control y prevención del lavado de activos adoptados al interior del continente responden a parámetros de aplicación internacional, por ejemplo, el principio conozca su cliente.
Ahora bien, la mayoría de los Estados miembros de la OEA — Organización de los Estados Americanos- han adoptado o se han comprometido a adoptar estas medidas en su ordenamiento jurídico interno. Estas recomendaciones internacionales se reflejan de manera integral en las distintas áreas de la legislación interna, que de algún modo se relacionan con el lavado de activos.

Las disposiciones que regulan el sistema financiero, el sistema penal y la estructura del apartado jurisdiccional en los países latinoamericanos, en cuanto hace al tema del lavado de activos, se encuentran adecuadas a los estándares internacionales en la materia.

La acción de las autoridades contra el lavado de activos tiene la mayor importancia en el entorno actual, en el cual se han modificado de manera sustancial las políticas contra la delincuencia. En este tiempo, se ha visto que una de las herramientas más efectivas contra el delito es perseguir la riqueza producto de las acciones cometidas por fuera de la ley, siendo la acción contra el lavado de activos una de las herramientas más efectivas.

Se requiere pues de un trabajo en conjunto para controlar el flujo de capitales entre los dis- 
tintos Estados, práctica habitual de las organizaciones criminales para eludir la acción de las autoridades.

La Organización de las Naciones Unidas ha definido el lavado de dinero como la ocultación o encubrimiento de la verdadera naturaleza, fuente, disposición, traslado o propiedad del producto del tráfico ilícito de drogas. El blanqueo incluye el traslado o la conversión de haberes, o de su producto, por cualquier medio, incluida la transmisión electrónica, con el objeto de hacerlos pasar por el producto de actividades licitas. En resumen, el lavado de activos es un delito proceso, por el cual una persona convierte o transforma el dinero obtenido de una actividad ilícita - por ejemplo, narcotráfico-, introduciendo dicho dinero al sistema bancario o una actividad empresarial - empresa fachada- con el objeto de convertir el dinero ilegal en dinero lícito.

Con la finalidad de combatir el lavado de dinero, los países en el mundo se han puesto de acuerdo en asumir compromisos e implementar "medidas de control" que se materializan en las normas de prevención de lavado de activos y financiamiento del terrorismo.

Por ejemplo, existen las 40 Recomendaciones del GAFI — Grupo de Acción Financiera Internacional-, las mismas que son lineamientos internacionales para combatir el lavado de dinero y sus modalidades en general, y las que en nuestro país se han implementado en el conjunto de normas relacionadas con la prevención del lavado de activos en distintos sectores. Por ejemplo, en primer lugar las Recomendaciones de GAFI establecen que los países deben implementar una Unidad de Inteligencia Financiera -UIFla cual deberá encargarse de procesar y analizar la información financiera detectando alguna irregularidad en la transmisión de capitales y comunicar al Ministerio Público para su pronta investigación; de igual forma GAFI establece que los países deben implementar un sistema de prevención de lavado de activos e incorporar en su organización un Oficial de Cumplimiento cuya función primordial sea la detección de ingreso de dinero ilícito en la organización, comu- nicando en su informe de manera confidencial al Directorio y al ente regulador para su posible investigación. Es decir, el Oficial de Cumplimiento es un profesional puesto en la organización por ley o norma legal, designado por el Directorio, quien sería el nexo de conexión entre el ente regulador - por ejemplo la Superintendencia de Banca y Seguros - SBS - y la entidad financiera.

\section{LA UIF - PERÚ ADSCRITA A LA SBS}

Durante el Gobierno de Alejandro Toledo, el Congreso de la República creó la Unidad de Inteligencia Financiera - Perú —UIF-Perú-, a través de la Ley 27693, publicada en el diario oficial el día viernes 12 de diciembre de 2002, otorgando el rango de personería jurídica de derecho público, con autonomía funcional, técnica y administrativa, encargada del análisis, el tratamiento y la transmisión de información para prevenir y detectar el lavado de dinero o activos, "con pliego presupuestal adscrito al Ministerio de Economía y Finanzas"; concepto posteriormente modificado por la Ley 28009 — del 21.06.2003-, y la Ley 28306 — del 29.07.2004-, las cuales establecen que la UIF tiene pliego presupuestal adscrito a la Presidencia del Consejo de Ministros -PCM-; y posteriormente, incorporándose la UIF a la Superintendencia de Banca y Seguros -SBS-, como unidad especializada.

El Reglamento primigenio de la Ley 27693 era el Decreto Supremo № 018-2006-JUS; actualmente derogado mediante Decreto Supremo $\mathrm{N}^{\circ} 020$ 2017-JUS, cuyo objetivo señalado en su artículo 1 es desarrollar las disposiciones contenidas en la Ley 27693 y sus modificatorias, para fortalecer la prevención, detección y sanción del lavado de activos y el financiamiento del terrorismo.

A continuación, por ejemplo en el país de Bolivia se le denomina Unidad de Investigaciones Financieras - UIF-, la cual es una entidad pública descentralizada con personalidad jurídica de derecho público, autonomía de gestión administrativa, financiera, legal y técnica, bajo tuición del Ministerio de Economía y Finanzas Públicas, y es la unidad encargada de recibir, solicitar, analizar y, en su caso, transmitir a las autoridades competentes la información ne-

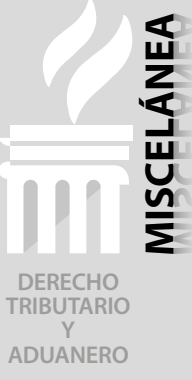


cesaria debidamente procesada vinculada a la legitimación de ganancias ilícitas y financiamiento.

En Argentina tiene el nombre de Unidad de Información Financiera - UIF- cuya función es el análisis, el tratamiento y la transmisión de información a los efectos de prevenir e impedir el lavado de activos provenientes de una serie de delitos graves, se encuentra bajo la jurisdicción del Ministerio de Justicia y Derechos Humanos de la Nación y depende presupuestalmente del Ministerio de Hacienda.

En Chile tiene el nombre de Unidad de Análisis Financiero - UAF - la cual es un servicio público descentralizado, con personalidad jurídica y patrimonio propio, que se relaciona con el Presidente de la República de Chile a través del Ministerio de Hacienda. Su objetivo es prevenir e impedir la utilización del sistema financiero, y de otros sectores de la actividad económica chilena, para la comisión de los delitos de lavado de activos y financiamiento del terrorismo.

En Colombia se llama la Unidad de Información y Análisis Financiero - UIAF-, la cual es una unidad administrativa especial del Estado colombiano, con personería jurídica, autonomía administrativa y financiera, de carácter técnico, adscrita al Ministerio de Hacienda y Crédito Público. Es el órgano de inteligencia financiera del con el fin de prevenir, detectar y luchar contra el lavado de activos y la financiación del terrorismo.

En Brasil la Agência Brasileira de Inteligência ABIN - abarca la producción de conocimientos de inteligencia para la Presidencia de la República y la participación en iniciativas interministeriales. La ABIN integra la Estrategia Nacional de Combate a la Corrupción y al Lavado de Dinero - ENCCLA, por su sigla en portugués-y tiene asiento en el Consejo de Control de Actividades Financieras - COAF-, unidad de Inteligencia financiera de Brasil, responsable por el proceso de obtención y análisis de informaciones y producción de conocimientos de Inteligencia necesarios al proceso de toma de decisiones del Poder Ejecutivo, además de actuar en la protección de las informaciones sensibles y estratégicas del Estado brasileño.

En México se le llama la Unidad de Inteligencia Financiera - UIF_ l la cual es una instancia de la Secretaría de Hacienda y Crédito Público de México encargada de prevenir y combatir los delitos de operaciones con recursos de procedencia ilícita y del financiamiento al terrorismo.

En Canadá se denomina Financial Transactions and Reports Analysis Centre, en español, Centro de Análisis de Informes y Transacciones Financieras de Canadá -FINTRAC_- unidad de inteligencia financiera de Canadá. Su mandato es facilitar la detección, prevención y disuasión del lavado de dinero y el financiamiento de actividades terroristas, al tiempo que garantiza la protección de la información personal bajo su control. Es una institución federal que forma parte de la cartera de finanzas y trabaja con sus socios del régimen contra el lavado de dinero y el financiamiento contra el terrorismo a nivel federal, provincial y municipal.

Como hemos podido apreciar, el tratamiento y creación de la UIF en la región no es absolutamente autónoma, son creadas por leyes y carecen de rango constitucional, y siempre dependen del brazo del Gobierno que alimenta estas instituciones.

Al respecto - debido a la gran importancia de la entidad para prevenir el lavado de dinero en un país- nosotros consideramos que la UIF-Perú debe ser un organismo del Estado autónomo e independiente elevado a rango constitucional, con presupuesto funcional y administrativo dotado de absoluta autonomía, sin ninguna dependencia directa o indirecta con las entidades u órganos del Gobierno como la Presidencia del Consejo de Ministros, que es un órgano directo de la Presidencia de la República, o del Ministerio de Justicia, y mucho menos incorporada a la SBS como "Unidad Especializada".

Por ejemplo, si se encuentra incorporada a la SBS, se duda mucho que pueda ejercer un control objetivo y eficiente contra los funcionarios del Estado y del Gobierno, lo mismo sucede en 
otros países donde la UIF no ha podido ejercer un control absoluto en materia de prevención de lavado de activos y corrupción contra altos funcionarios y líderes políticos. Mucho se habla del caso Odebrecht pero nadie ha preguntado cómo se han realizado estas transferencias de capitales y sus respectivos sustentos, cómo ha fallado el sistema de prevención en las entidades financieras supervisadas o la lentitud de la UIF para ejercer su investigación. En consecuencia, consideramos que la UIF no ha estado preparada para asumir tal responsabilidad, no porque quiera evadirla, sino porque que el Estado - Gobierno y Congreso- no la ha dotado de absoluta autonomía para su eficaz ejercicio y control.

La razón de ser de la UIF es ejercer la prevención, control e investigación en materia de lavado de dinero y activos en un país, quien luego de tener una sospecha razonable, remitirá su informe confidencial y elevará los partes al Ministerio Público para su correspondiente investigación penal; pero si ésta se encuentra adscrita a un ente del Gobierno dudamos mucho que ejerza su función con total independencia y objetividad.

Hacemos una pregunta: ¿alguno de ustedes ha escuchado hablar de la UIF-Perú en algún medio de comunicación? No tenemos alcances de noticias de si la UIF-Perú ha comunicado al Ministerio Público algún tema sospechoso de lavado de dinero. ¿Cuántos funcionarios del Estado, líderes políticos o empresarios son o vienen siendo investigados por estos delitos ante la fiscalía?

Por lo tanto, sostenemos que la UIF-Perú debe ser una entidad del Estado autónoma con presupuesto independiente y sobre todo con capacidad de investigar actividades sospechosas de presuntos delitos de lavado de dinero, inclusive a altos funcionarios del Estado, ministros, asesores de la presidencia, congresistas, etc., cuya actividad y/o función involucre a los altos funcionarios del Estado.

Cuando fuimos Oficial de Cumplimiento en una empresa, recibimos por correo una invitación de la SBS para asistir a un evento desayuno so- bre implementos para mejorar el sistema de prevención de lavado de activos. Escuchamos a los expositores, y, luego, en nuestra mesa los consultores, nos pidieron que llenemos unos papeles de trabajo para su retroalimentación, todos expusieron y comentaron sus puntos de vista para "mejorar" el sistema de prevención; cuando llegó nuestro turno fuimos muy directos en decir que estos eventos de consultoría no serán eficientes y no servirán de nada si es que la UIF-Perú sigue incorporada a la SBS. Nuestro punto de vista es el mismo, consideramos que la UIF-Perú debe ser una entidad del Estado autónoma sin depender jerárquicamente de ningún aparato del Estado y sobre todo debe ser elevado a rango constitucional por iniciativa del Congreso.

\section{LA DEDICACIÓN NO EXCLUSIVA}

De otro lado, para aquellos que han ejercido el cargo, sabrán que las funciones de un Oficial de Cumplimiento son diametralmente distintas a las funciones de un Asesor Legal. Este último tiene funciones estrictamente jurídicas: por ejemplo, revisión de contratos, mitigar riesgos legales para la sociedad, elaboración de oficios, elaboración y contestación de demandas judiciales, recursos impugnatorios judiciales y administrativos en defensa de la sociedad, acuerdos de transacciones extrajudiciales, revisión de poderes, elaboración de actas, entre otros de índole legal. Sin embargo, las empresas por reducir costos - y también porque la norma lo permite- contratan abogados que ejercerán la función de Oficial de Cumplimiento y a la vez la de asesor legal, sabiendo que a la larga traerá consecuencias nefastas para la sociedad y para el profesional mismo.

En primer lugar, por norma legal, el Oficial de Cumplimiento depende "jerárquicamente del Directorio", por lo tanto, deberá reportar directamente al Directorio; sin embargo, en el caso del Asesor Legal no sabemos si la estructura orgánica se encuentra diseñada para reportar al Directorio.

Muchas veces recibe consultas de diversas áreas de la empresa, por lo tanto, su función se cir- 
cunscribe de soporte legal o asesoría legal a los demás gerentes de la empresa, recibiendo muchas veces consultas de otras áreas en el sentido que no se ha implementado un mecanismo de procedimiento de consultas legales idóneas.

Como se habrán podido dar cuenta, esta función de asesoría y soporte dista de la función stricto sensu del Oficial de Cumplimiento, quien está obligado por ley a reportar directamente al Presidente del Directorio mediante los informes semestrales o trimestrales internos los cuales son estrictamente "confidenciales". Es decir, las normas han determinado que el Oficial de Cumplimiento sea el nexo entre el ente regulador y la sociedad - Banco, sociedad minera, casino, etc.- y sus dueños, directores o accionistas. Por ejemplo, no existe una norma legal que obligue o imponga a un gerente de una empresa para sea el nexo o canal con el Ente Regulador.

Por lo tanto, el asumir una doble función dedicación no exclusiva - no ayuda al fortalecimiento y control de medidas antilavado; $y$, muchas veces por asumir una doble función, no se realiza el control eficiente de implementación de Políticas de Prevención del Lavado de Activos y Financiamiento del Terrorismo -en adelante, "PLAFT" - asumiendo la sociedad un riesgo innecesario, tanto los Directores como accionistas de la sociedad, ante eventuales inspecciones del ente regulador al verificar que la documentación en materia de prevención no se encuentra ordenada, siendo insuficiente e incompleta debido a la doble función, acarreando ésta una eventual sanción razonable.

Sostenemos que la función del Oficial de Cumplimiento debe ser estrictamente exclusiva, y la norma no debe permitir que pueda realizar una función a dedicación no exclusiva, pues trae consecuencias nefastas para la función del Oficial de Cumplimiento, en el sentido que debe enfocarse a detectar posibles ingresos de dinero ilícito en la organización y comunicar lo más pronto posible al Directorio y al ente regulador; sin embargo, la función adicional que asume de asesor legal posiblemente no le permitirá identificar oportunamente dichas actividades lo que pone en riesgo la actividad de la empresa. En consecuencia, hemos identificado una falla en el sistema de prevención que tiene que ser modificada.

Hace unos días nos llamaron de una entidad financiera quienes estaban interesados en realizar una consultoría. Lo primero que hicimos es preguntar al gerente general si el Oficial de Cumplimiento desempeña una función "a dedicación no exclusiva", nos respondió que no, que el Oficial de Cumplimiento desempeña una función a dedicación exclusiva. Aceptamos reunirnos con ellos porque el trabajo de implementación de control es más fácil y eficiente.

Según nuestra experiencia, cuando estuvimos capacitando una entidad financiera, el Oficial de Cumplimiento estaba haciendo su labor, pero se notaba que su mente estaba en otra reunión. Le preguntamos qué sucedía, y nos indicó que en unos minutos lo llamaría el gerente para revisar una contestación de demanda judicial cuyo plazo vencía ese día, con lo cual tuvo que retirarse y abandonar la capacitación, transmitiendo un mensaje al equipo de trabajo y a la organización que el tema de prevención y cumplimiento no era importante para él.

Imagínese usted que el arquero Iker Casillas del Real Madrid tenga a su cargo la función del zaguero Sergio Ramos, es decir, sería arquero y defensa simultáneamente, las consecuencias quizá las podemos adelantar. Imaginemos por un momento que el Primer Ministro sea a su vez Ministro de Salud, ¿ustedes creen que podría desempeñar una buena tarea pública y tomar decisiones acertadas y urgentes?

\section{SUPUESTA AUTONOMÍA}

De igual forma, para asumir una función eficiente el Oficial de Cumplimiento no solamente debe dedicarse a una función exclusiva, sino también debe gozar de una absoluta autonomía real.

Cuando un Oficial de Cumplimiento se incorpora recientemente a sus labores tiene que firmar una serie de documentos en calidad de declaración jurada - porque así lo establece la norma y no tiene otra salida-, entre ellos, señala y 
firma expresamente que "el Oficial de Cumplimiento goza de absoluta autonomía e independencia en el ejercicio de las responsabilidades y funciones, y que depende jerárquicamente dentro del organigrama funcional directamente del directorio". Sin embargo, advertimos que no es posible gozar de una autonomía absoluta cuando el Oficial de Cumplimiento es "a dedicación no exclusiva", - porque la norma lo permite-, pues en este caso también es asesor legal quien no goza de autonomía absoluta en sus funciones, y ni modo que diga al momento de incorporarse a su labor que no goza a autonomía absoluta y se niegue a firmar el referido documento. Finalmente, debido a la falta de autonomía real, muchas veces los informes del Oficial de Cumplimiento - que son revisados y/o corregidos previamente por el Gerente General- carecen de valor para el debido control del lavado de dinero, transmitiendo un informe débil que satisfaga los intereses de la empresa.

Por ejemplo, en las transacciones financieras realizadas en el caso de la brasileña Odebrecht, sus vinculadas, y proveedores que giran a su alrededor, los Oficiales de Cumplimiento tenían la obligación y deber de identificar el origen de los fondos y las cuentas bancarias, los beneficiarios o destinatarios reales, cruzar información con otros bancos, revisar los datos de los apoderados, la triangulación del dinero, la naturaleza real de los contratos y operaciones, para determinar si son o no ficticios, solicitando la información que considere necesaria para el debido control. Sin embargo, muchas veces el requerimiento de información es bloqueado por el mismo Gerente General —que no es experto en cumplimiento-, por temor a incomodar al "inversionista-cliente" con preguntas inoportunas, y retire su dinero y lo lleve a otro banco de la competencia; esto se debe a la falta de autonomía real del Oficial de Cumplimiento para el efectivo control del lavado de activos. No se trata de convencer a los directivos del banco para que requieran información, sino el Oficial de Cumplimiento debe estar dotado y empoderado de una autonomía absoluta que le permita ejercer su función para el control de lavado de activos y poder identificar el origen de los capitales ilícitos.
Los directivos deben entender que todo el estudio de prevención de lavado de activos gira en un solo concepto universal: el delincuente tiene la intención planificada de aparentar un negocio lícito, por lo tanto, detrás de una operación se oculta una estrategia diseñada para convencer una apariencia de un negocio lícito, cuando realmente el delincuente se encuentra lavando el dinero, haciendo una triangulación y operación ficticia o de fachada, por ejemplo, para una operación ilícita entrega un nombre o empresa de un tercero para no dejar rastros que vinculen con el acto ilegal.

Si una entidad bancaria, financiera o sociedad agente de bolsa - cuya actividad principal es el financiamiento-, tiene un cliente del rubro construcción y ésta para operar tiene distintas sucursales o empresas creadas bajo una misma matriz, y a su vez los fondos provienen del extranjero: por ejemplo, la empresa "Constructora Juanitos S.A.C.", tiene a su vez o es dueña de la sub empresa "Juanitos ABC S.A.C", "Juanitos DEF S.A.C", "Juanitos OPQ S.A.C.", y "Juanitos

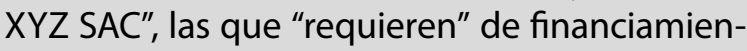
to para la edificación del proyecto.

Aquí, el Oficial de Cumplimiento deberá solicitar al cliente la información y/o documentación que sustente dicha operación, y verificar el origen de los fondos, cruzar información e identificar al beneficiario final real. Sin embargo, como ya hemos referido, muchas veces el Oficial de Cumplimiento solicita dicha información, la cual es negada previamente por su propio Gerente General y/o el Directorio de la sociedad, obstaculizando la función del Oficial de Cumplimiento debido a que la norma lo permite.

\section{ANTICIPACIÓN DEL DIRECTORIO}

Hace unos años tuvimos la oportunidad de trabajar en un Operador Logístico. El gerente general realizó una consulta legal, sobre si era necesario o no implementar un Sistema de Prevención PLAFT en la organización, y la respuesta a la consulta fue que no existe una obligación legal. Por lo tanto, no se implementó dicho sistema. Algún tiempo después, tomamos conocimiento que la empresa colombiana BIOMAX

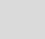


- dedicada a la distribución de combustibles derivados de petróleo- tenía implementado el Sistema de Prevención SARLAF — sistema de administración de riesgos de lavado de activos y financiamiento del terrorismo-, la cual era encabezada por su Oficial de Cumplimiento. Este sistema cuenta con políticas, controles, y procedimientos establecidos a fin de prevenir oportunamente el uso o la relación de la compañía con personas y/o organizaciones con vínculos en actividades ilícitas como el lavado de activos y financiamiento del terrorismo. Asimismo, este sistema tiene como propósito la identificación y el reporte de las operaciones que pretendan permear la organización. Pero lo más relevante era que, aunque no fuera una obligación legal impuesta, la alta gerencia y el Directorio se habían anticipado a la decisión oportuna de implementar dicho Sistema de Prevención aunado a un Código de Ética, porque sabían que con ello fortalecerían las barreras de entradas de capital ilícito a la organización.

Como se puede apreciar, ante la deficiente regulación, los directivos - prudentes en los negocios - tienen la obligación de anticiparse y tomar la iniciativa para prever en el futuro una contingencia que involucre o ponga en riesgo la marcha y actividad económica del negocio, implementando mecanismos de control para evitar el ingreso de dinero ilícito en la organización.

En consecuencia, la responsabilidad directa de toma de decisiones en implementar eficientemente un Sistema de Prevención PLAFT en la organización recae en el Directorio y el gerente general, todo ello con el fin de prevenir el lavado de dinero en una determinada organización; no hacerlo es una omisión considerable que podría tener consecuencias nefastas de índole penal para sus dueños, sin advertir desde ya los riegos de reputación.

\section{REMOCIÓN DEL OFICIAL DE CUMPLI- MIENTO}

Sobre la remoción del Oficial del Cumplimiento, las normas PLAFT tienen una gran deficiencia.

Un Oficial de Cumplimiento supuestamente tiene cargo de confianza; reporta, también supuestamente- directamente al Directorio, cuenta con autonomía absoluta y presupuesto para el adecuado desempeño de sus funciones. La norma PLAFT también le otorga rango de calidad de gerente; por lo tanto, deberíamos entender que sus funciones y categoría laborales obedecen al de un cargo de confianza; sin embargo, no es tan sencillo como parece ser.

En el eventual caso que la gerencia general no se encuentre conforme con el trabajo y desempeño del Oficial de Cumplimiento, tendrá que invitarlo a renunciar y/o celebrar una resolución de contrato laboral por mutuo disenso, esto último con la finalidad de evitar que el Banco o Sociedad Agente de Bolsa regulada por la SBS y SMV, respectivamente, tengan la obligación de elaborar un Informe y remitir a la UIF-Perú.

En consecuencia, si el ejecutivo Oficial de Cumplimiento encuentra injusta su destitución que puede darse por motivos personales-, con todo derecho puede negarse a firmar la carta de renuncia y/o firmar la resolución del contrato laboral por mutuo disenso, con lo cual aceptaría implícitamente parte de su culpa en el desarrollo de sus funciones. No obstante, sostenemos que si no existen fundamentos para la remoción del Oficial de Cumplimiento, la sociedad tendrá que elaborar obligatoriamente su Informe en el cual sustente su remoción, Informe que deberá ser "aprobado" por el Directorio y el gerente general en su conjunto, que fundamente las razones que justifican tal medida. La remoción y el informe que la sustente serán comunicados por el sujeto obligado a la UIF-Perú y a la SMV, dentro del plazo de cinco días hábiles de adoptada la decisión.

Hasta aquí parece no haber ningún inconveniente en su regulación; sin embargo, lo existe y es de mucha gravedad y perjuicio para el Oficial de Cumplimiento, ya que la UIF-Perú no ha previsto tal injusticia. Es decir, ante la negación laboral del Oficial de Cumplimiento de firmar la carta de renuncia y/o el documento por mutuo disenso, el gerente general tendrá que comunicar inmediatamente tal situación para que el Directorio tome acciones y apruebe el Informe que susten- 
te tal medida, el mismo que deberá ser aprobado - no olvidemos como señala la norma- por el Directorio y por el gerente general, la misma que deberá ser comunicada a la UIF-Perú y al ente regulador dentro del plazo de ley.

Sin embargo, luego de que ya el Oficial de Cumplimiento ha sido destituido de su cargo con el envío de la carta de despido o retiro de confianza, la sociedad tiene un plazo de cinco días para elaborar el Informe que sustente tal medida. Pues bien, este Informe elaborado por el gerente general y sus colaboradores puede estar lleno de defectos refiriéndose a la conducta y capacidad del Oficial de Cumplimiento, debido a que la ruptura laboral fue ocasionada por motivos personales y no propiamente de sus funciones.

Pero nuestra preocupación va más allá, en el sentido que si la UIF-Perú, la entidad y santidad que tiene la función de velar por el adecuado cumplimiento del sistema de prevención de lavado de activos, no verifica, ni cruza información sobre el contenido del referido Informe; y más aun, mucho menos la SBS y la SMV se toman el trabajo de revisar y verificar su contenido, solicitando de oficio información documentada y probanza que ampare dicha remoción, al parecer los entes reguladores se conforman con recibir el citado Informe de remoción sin verificar el contiendo de la misma, con la cual se ve perjudicada la imagen y rol profesional del Oficial de Cumplimiento, al dar por entendidas y satisfechas las supuestas razones que motivaron su destitución puestas en el Informe, sin verificar su autenticidad y prueba documental.

Desde aquí invocamos a los entes reguladores a tomar más acción y control cuando reciban estos tipos de Informes que pudieran están causando un perjuicio al Oficial de Cumplimiento y también al sistema de prevención nacional, teniendo presente que es su obligación y competencia velar por el adecuado cumplimiento del sistema de prevención de lavado de activos, así como verificar el cumplimiento, por parte de las sociedades autorizadas, de la normatividad aplicable al desarrollo de sus funciones, dentro del marco establecido por las Normas para la
Prevención del Lavado de Activos y Financiamiento del Terrorismo.

\section{INFORMES CONFIDENCIALES Y RESER- VADOS}

Otro tema muy importante que no se comenta y que resta valor al trabajo de prevención es la "Confidencialidad de la información en los Informes". Por ejemplo, la norma sobre prevención de lavado de activos establece que en caso el Oficial de Cumplimiento comunique a la UIF-Perú un Reporte de una Operación Sospechosa - ROS - éste deberá ser con carácter confidencial y reservado. Únicamente el Oficial de Cumplimiento, o de ser el caso el Oficial de Cumplimiento alterno, puede tener conocimiento del envío del ROS.

Sin embargo, consideramos que la norma se contradice al establecer que en los informes trimestrales y semestrales elaborados por el Oficial de Cumplimiento dirigidos al Presidente del Directorio y a la UIF-Perú se indica que este informe debe contener el número de ROS enviado a la UIF-Perú.

Al mismo tiempo la norma permite la creación de un Comité PLAFT facultativo; sin embargo, creemos que este Comité no ayuda ni fortalece el sistema de prevención en el sentido que el "Comité" se encuentra integrado por profesionales no expertos en materia de prevención, ni mucho menos tienen conocimientos de derecho penal respecto a la magnitud y gravedad del delito de lavado de dinero y sus consecuencias para la sociedad.

Asimismo, la norma también, equivocadamente creyendo que fortalece el sistema de prevención, señala que dentro de las funciones del Oficial de Cumplimiento, entre otras, se encuentran, analizar las operaciones inusuales detectadas con asesoría del Comité, a fin de determinar las operaciones que podrían ser calificadas como sospechosas; es decir, y como se puede apreciar, la norma se contradice en el sentido que en el Comité se va a discutir las operaciones inusuales para ser consideradas como sospechosas teniendo pleno conocimiento desde ya el Comité de cierta

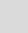


información que es estrictamente confidencial y que solamente atañe al Oficial de Cumplimiento y la UIF-Perú —a nadie más-; debiendo tener presente que la norma establece que dicha información es de absoluto carácter confidencial y reservado, siendo el Oficial de Cumplimiento el único que debe tener conocimiento del mismo y será responsable de comunicar tal hecho a la UIF-Perú, pudiendo generarse con el informe consecuencias penales que ameriten una pronta investigación.

No obstante, dentro de las funciones del Oficial de Cumplimiento la norma PLAFT absurdamente lo obliga a: "analizar las operaciones inusuales detectadas, con la asesoría del Comité para la Prevención del Lavado de Activos y del Financiamiento del Terrorismo si lo hubiere, con la finalidad de determinar las operaciones que podrían ser calificadas como sospechosas".

\section{REFLEXIONES FINALES}

En resumen y como reflexiones finales, en los últimos años hemos visto como las autoridades se han esforzado en legislar con el fin de erradicar y mitigar el lavado de dinero en el Perú; no obstante, consideramos que si el objetivo es "fortalecer" el sistema de prevención en el país debemos modificar la legislación en las anotaciones identificadas anteriormente:

1. La autonomía absoluta de la UIF-Perú elevado a rango Constitucional;

2. La dedicación exclusiva del Oficial de Cumplimiento;

3. La autonomía real y absoluta del Oficial de Cumplimiento en sus funciones;

4. Ante la deficiente regulación PLAFT, el Directorio deberá anticiparse para prever en el futuro una contingencia que ponga en riesgo la marcha del negocio, implementando mecanismos de control para evitar el ingreso de dinero ilícito en la organización, y al mismo tiempo, evitar consecuencia penales;

5. El Informe de remoción del Oficial de Cumplimiento deberá ser verificado por el ente regulador y la UIF-Perú, quienes dentro de su obligación y competencia podrán solicitar documentación al respecto para el control del sistema de prevención y;

6. El Informe confidencial y reservado que comunica el Oficial de Cumplimiento a la UIF-Perú debe ser de conocimiento único del Oficial de Cumplimiento. 